Rev. Biol. Trop. 46(4): 1033-1037, 1999

www.ucr.ac.cr www.ots.ac.cr www.ots.duke.edu

\title{
COMUNICACIÓN
}

\section{Historia natural y presencia de la "planta insectívora" Drosera capillaris (Droseraceae) en Costa Rica}

\author{
Luis D. Gómez P.1 y Jorge Gómez-Laurito²
}

1 Academia Nacional de Ciencias, Apartado 1357-2050, Costa Rica; 2 Herbario, Escuela de Biología, Universidad de Costa Rica, 2060 San José.

1, 2 Investigadores Asociados, Field Museum of Natural History, Chicago, IL., Proyecto Flora costaricensis.

Recibido 19-I-1998. Corregido 18-IX-1998- Aceptado 5-X-1998

\begin{abstract}
The family Droseraceae is reported for the first time from Costa Rica represented by Drosera capillaris Poiret. The species grows in vernal pools of hyperhumid savannahs of the Parque Nacional Amistad, Puntarenas Province. The population was small, of some 30 individuals that reduce dipterans to empty exoskeletons in $82 \mathrm{hr}$. Glandular secretion in higher when humidity is high and light is low. To date, the nearest locality reported for this species was Mexico.
\end{abstract}

Key words: Droseraceae, Drosera capillaris, Costa Rica, floristics.

La familia Droseraceae consta de cuatro géneros, Aldrovanda Monti, Dionaea Ellis, Drosophyllum Link, monotípicos y con distribuciones geográficas relativamente restringidas, y Drosera L., con un centenar de especies de amplia distribución en zonas templadas y tropicales de ambos hemisferios aun cuando la región australiano-neozelandesa concentra un alto porcentaje de esas especies (Diels 1906, Kondo y Kondo 1983, Lloyd 1942).

Todas las droseráceas pertenecen a las llamadas "plantas insectívoras", por tener en sus hojas pelos glandulares que segregan mucílagos que atrapan insectos. En Aldrovanda y en Dionaea, las hojas enteras funcionan como trampas mientras que en los otros géneros la captura es de tipo adhesivo aun cuando los pelos son irritables. Algunos pelos son digestores, otros son glandulares (coléteros) que segregan sustancias ergástricas. Los marginales laminares no son, propiamente pelos, sino lóbulos foliares, así co- mo no son tricomas los otros glandulares vascularizados, sino proyecciones subepidérmicas.

Se conocían hasta la fecha varias especies de Drosera de México, savanas del EN de Nicaragua y Panamá pero no de Costa Rica. Aquí se registra por primera vez la siguiente especie de droserácea:

\section{Drosera capillaris Poir., Encycl. VI. 299.1804.}

Plantas herbáceas, acaulescentes, arrosetadas, con raíces fibrosas y estoloníferas. Hojas largamente espátulo-redondeadas, simples $20-30 \mathrm{~mm}$ de largo; estípulas intrapeciolares $2.5-4.5 \mathrm{~mm}$ de largo, 3 (raro 4)-partidas, soldadas casi hasta los senos de los lóbulos o fimbrias, que son largamente aguzadas, con dentículos marginales escasos y dispersos; peciolo diferenciado, $10-14(16) \mathrm{mm}$ de largo, 1 -1.2 $\mathrm{mm}$ de ancho en su parte media, en la base con seudoalas formadas por tejido de estípulas 
intrapeciolares, ensanchándose ligeramente en la base de la lámina foliar, en corte transversal dorsiventralmente aplanado, con tres hacecillos vasculares, con pelillos aciculares cortos, de 1 -3 células, distantes en los bordes, más frecuentes en terciodistal, haz con pelillos glandulares sésiles y glandulosos fungoides (coléteros) dispersos, envés con pelos aciculares pluricelulares sobre los haces vasculares y dirigidos hacia el ápice de la lámina, adpresos.

Lámina ampliamente abovada, 3.5 -4.3 mm de largo, 3.8 -4.5 mm de ancho, con $19-25$ pelos tentaculares marginales, glandulares, de 2.3 3.1 (-4) mm de largo, ápice magenta, clavulado. Envés glabrescente, con pocos pelos no glandulares, aciculares, pluricelulares, sobre las venas; haz con numerosos pelos glandulares, capitados, los mediales cortos, $0.6-1.3 \mathrm{~mm}$, los infra y submarginales más largos, $1.5-1.8(-2)$ mm y 2 -3.5 $\mathrm{mm}$, respectivamente. Escapos $1-2$ por planta, capaces de proliferar apicalmente, $12-32 \mathrm{~cm}$ de largo, con numerosos y dispersos pelillos glandulares coléteros y glandulares sésiles, y algunas brácteas foliosas, lanceoladas, $0.7-1.2 \mathrm{~mm}$ de largo, $0.1-0.2 \mathrm{~mm}$ de ancho, enteras, glabras, verdes. Inflorescencia cimosa, de 12 -26 flores en los dos tercios distales, distantes entre sí 5 -9 mm. Pedicelos de $2-2.8 \mathrm{~mm}$ de largo, con pelos glandulares sésiles y fungoides. Sépalos 5, 1.5 $2 \mathrm{~mm}$ de largo, $0.7-0.8 \mathrm{~mm}$ de ancho, oblanceolados, soldados hasta su parte media, hacia el ápice irregular y escasamente aserrados, densamente vestidos con pelillos glandulares sésiles. Pétalos 5, oblongo-espatulados, 5 -6 × 3 -4.2 $\mathrm{mm}$, roseo-liláceos, secando magenta, persistentes. Estambres 10, polen en tétradas. Estilos 3, bipartidos. Ovario 5 -carpelar, 1-locular, con 3 placentas parietales que subtienden numerosos rudimentos seminales. Semillas inmaduras café avellana, en la madurez café oscuro o casi negras, elíptico-fusoideas, con 14-16 quillas longitudinales y numerosos procesos transversales, siendo así alveo-foveoladas, de 550 -650 $\mu \mathrm{m}$ de largo, en su parte media de $200 \mu \mathrm{m}$ de diámetro. Fruto una cápsula lóculo-poricida, dehiscente por 5 valvas. Florece de abril a mayo y madura los frutos en julio. (Figs. 1, 2a, 2b, 3).

En lagunas vernales, con Sphagnum y otros briófitos, Bulbostylis sp., Scirpus sp., Anoxopus sp. Parque La Amistad-Talamanca, vertiente occidental, a $750 \mathrm{~m}$ slm, Provincia de Puntarenas. L.D. Gómez 25914, USJ, F, PMA. Una cepa viva, en cultivo, en el Jardín Botánico R. \& C. Wilson, Las Cruces, Coto Brus, $\mathrm{N}^{\circ}$. 96.006. La única población observada es de unas 30 plantas.

La especie se conocía del sureste de E.E.U.U. pero Wood (1960) indicó su extensión hasta México. Su congénere geográficamente más cercano es Drosera cayennensis Sagot ex Diels $(=D$. panamensis

Correa \& Taylor, Ann. Missouri Bot. Garden 63: 390. 1976) cuyo ámbito es Panamá, Colombia, Venezuela y Guyana (Duno \& Culham 1995).

Las plantas costarricenses muestran un trofotactismo marcado. Si se coloca una mosca drosofílida exalada, los pelos tentaculares cortos son los primeros en dirigirse hacia la presa, seguidos por los pelos tentaculares superficiales y luego los marginales hasta que el ápice de la lámina se incurva sobre el insecto por unas horas. El proceso se observa en $24 \mathrm{hr}$. (los dípteros son exosqueletos vacíos de fluídos en 82 horas), y parece ser más activo durante la noche que a plena luz diurna. La secresión de las glándulas es mayor con alta humedad relativa y un bajo nivel fótico, lo que sugiere una adaptación a la captura de pequeños artrópodos nocturnos, lo que requiere observación directa en el hábitat natural. Los pelos glandulares coléteros (Hanstein 1868, Foster 1949), fungoideos, y los sésiles presentan un contenido opaco, resinoide, cuya función no es evidente.

Agradezco Betty Strack, Field Museum of Natural History, Chicago, por la microscopía electrónica de las semillas, a Mireya Correa, Universidad de Panamá, por su valioso comentario al manuscrito y a M. Hasbrouck por la Fig. 1. 


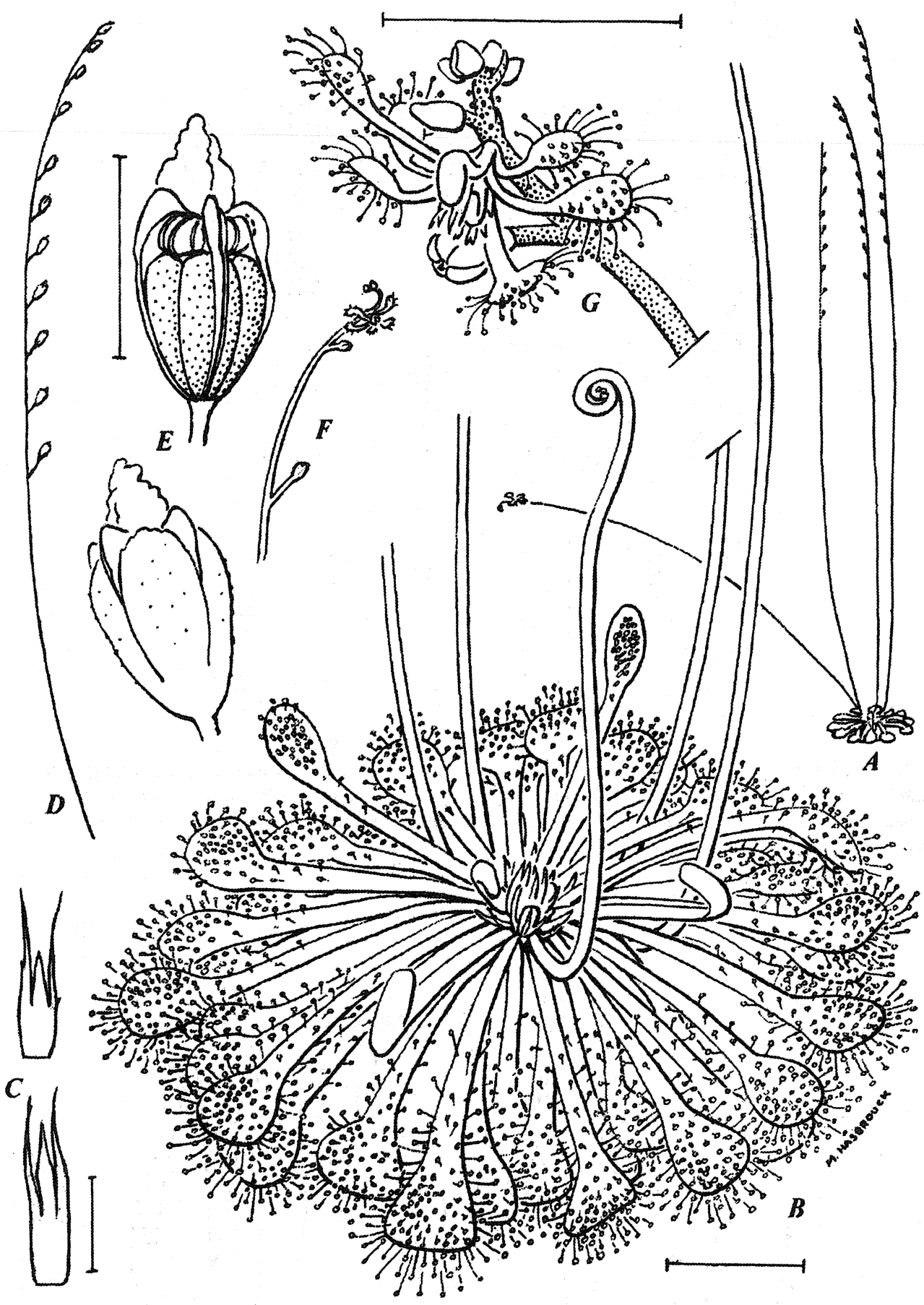

Fig. 1. a- hábito general, b- detalle de la roseta mostrando un escapo y hojas juveniles circinadas (Barra $=1 \mathrm{~cm})$, c- estípulas intrapeciolares $(B a r r a=2 \mathrm{~mm}), \mathrm{d}$ - inflorescencia cimosa, $\mathrm{e}$ - fruto inmaduro e inmediatamente antes de dehiscencia $($ Barra $=2 \mathrm{~mm}), \mathrm{f}, \mathrm{g}$ - detalle del ápice prolífero del escapo $($ Barra $=1 \mathrm{~cm})$. 


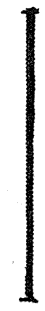
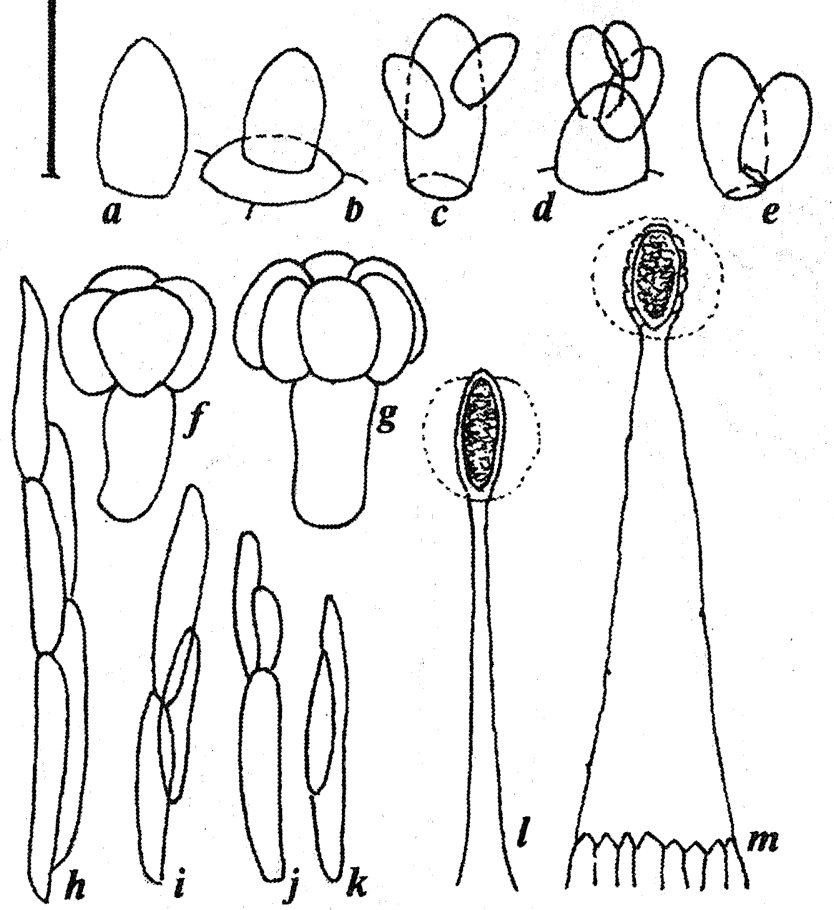

Fig. 2a. Tipos de pelos. a - e, pelos glandulares sésiles $(<0.3 \mathrm{~mm}) ; \mathrm{f}-\mathrm{g}$, pelos glandulares, coléteros, fungoideos $(0.6$ $1.2 \mathrm{~mm}$ ) f- pelo acicular corto ( $\leq 1 \mathrm{~mm}$ ); $\mathrm{h}-\mathrm{k}$, pelos aciculares largos (>l mm); l- pelo tentacular sub- o inframarginal, superficial, con una base de 3 - 5 células, $3-3.5 \mathrm{~mm}$; m- pelo glandular marginal (lóbulo foliar) con base de 6 - 13 células, $>3 \mathrm{~mm}$. (La barra $=1 \mathrm{~mm}$, para $\mathrm{a}-\mathrm{k}$ ).

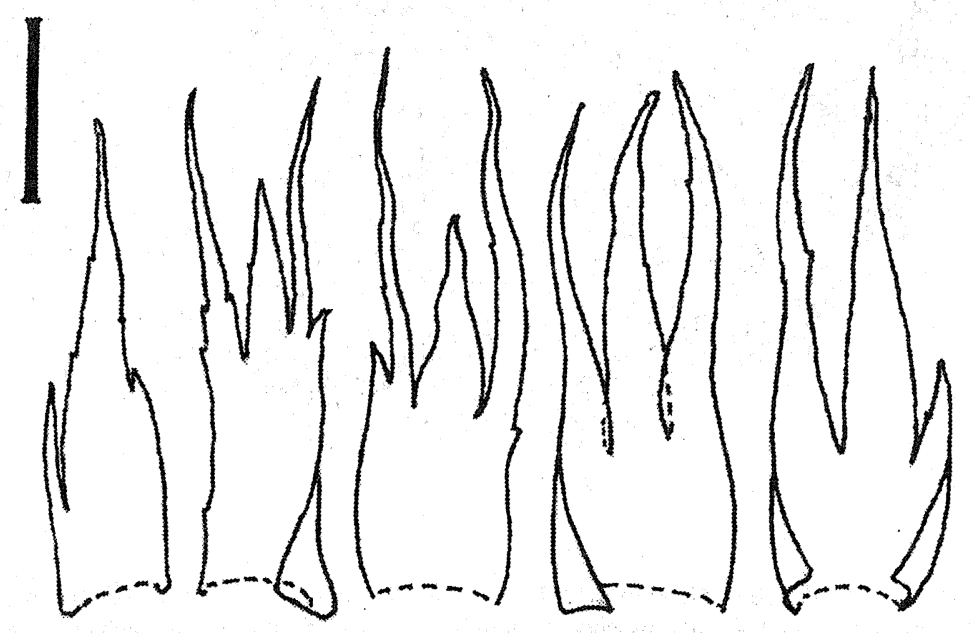

Fig. 2b. Formas de las estípulas intrapeciolares. Barra $=2 \mathrm{~mm}$. 

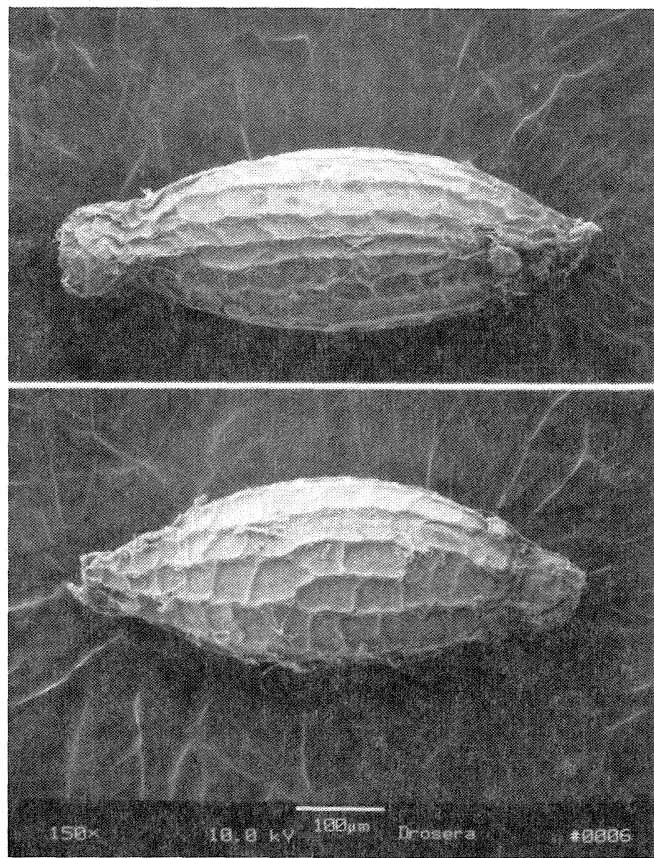

Fig. 3. Semilla vista al MEB. Barra $=100 \mu \mathrm{m}$.

\section{REFERENCIAS}

Diels, L. 1906. Droseraceae. Das Pflanzenreich 26: 1-136.

Duno, R. \& Culham, T. 1995. Dos especies nuevas del género Drosera (Droseraceae) en Venezuela y otros comentarios taxonómicos. Novon 5: 241245.

Foster, A.S. 1949. Practical Plant Anatomy. van Nostrand, Nueva York. p. 72-77.

Hanstein, J. 1868. Ueber die organe der Harz und Schleimabsonderung in dem Laubknospen. Bot. Zeit. 26: $697-713$.

Kondo, K. \& M. Kondo. 1983. Carnivorous plants of the world. Ienohikari, Tokio. $230 \mathrm{p}$.

Lloyd, F. E. 1942. The Carnivorous Plants. Ronald, Nueva York. $352 \mathrm{p}$.

Wood, C.E. 1960. The genera of Sarraceniaceae and Droseraceae in the southeastern United States. J. Arnold Arb. 41: 152-163. 